\title{
Alkylphosphatintoxikation - Das sollten Sie wissen für die Ergänzungsprüfung
}

Rico Kuhnke, Thomas Ahne
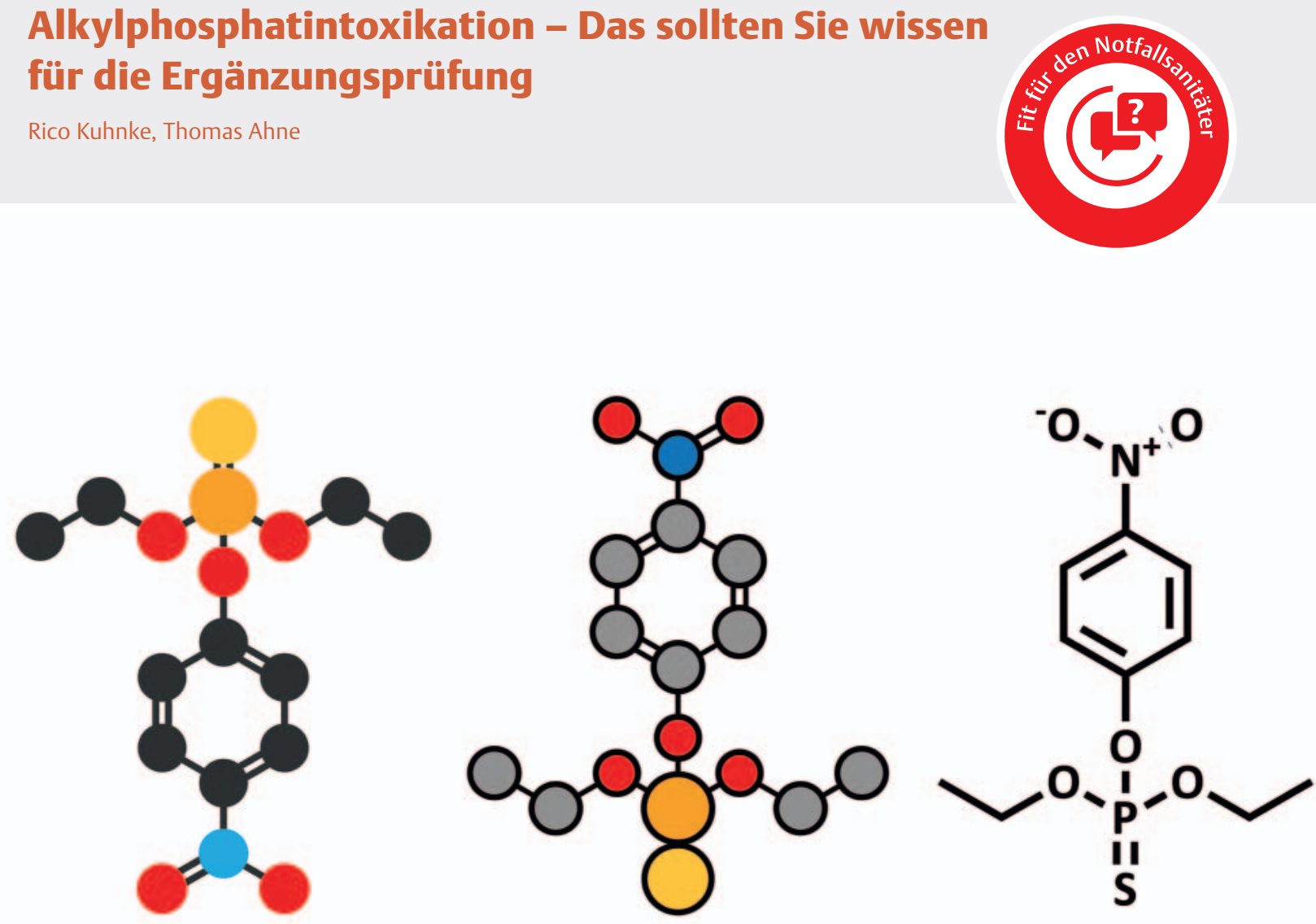

Quelle: molekuul.be/stock.adobe.com

retten! macht Sie fit für den Notfallsanitäter: In jeder Ausgabe arbeiten wir anhand eines Fallbeispiels einen interessanten Einsatz algorithmenkonform auf. Anhand von exemplarischen Fragen zu erweiterten Notfallmaßnahmen, Kommunikation und Rahmenbedingungen können Sie sich auf die Ergänzungsprüfung vorbereiten - egal, in welchem Bundesland Sie arbeiten.

\section{Fallbeispiel}

\section{Einsatzmeldung}

An einem schönen Frühsommertag wird der Rettungswagen zu einem Bauernhof gerufen. Die Leitstelle meldet einen Verdacht auf einen Suizidversuch.

\section{Situation vor Ort}

Auf dem Hof wird das Team bereits von einer jungen Frau erwartet. Sie ist aufgelöst, zeigt auf einen Schuppen in der Nähe des Gemüsegartens. Dort habe sie ihren
Ehemann bewusstlos auf dem Boden liegend gefunden. Im Schuppen habe es beißend nach Knoblauch gerochen. Sie habe ihren Mann sofort aus dem Schuppen gezerrt und in die Seitenlage gelegt.

Sie befürchtet, ihr Mann habe sich das Leben nehmen wollen. Vor einem Jahr hätten sie den Bauernhof gekauft und die aktuelle finanzielle Lage sei katastrophal. Noch gestern habe ihr Mann davon gesprochen, dass er am liebsten tot wäre. Danach hätten sie heftig miteinander gestritten, aber sie habe nie geglaubt, dass ihr Mann dies wirklich ernst gemeint habe. 
Sie führt das Team zu einer Stelle in der Nähe des Schuppens. Auf dem Boden liegt ein ca. 40-jähriger Mann, aus dem Mundwinkel läuft bläulicher Speichel.

\section{Eigenschutz}

Angesichts der Erzählungen der Frau und der vorgefundenen Situation (S-cene, S-ituation) erkennt das Team eine mögliche Eigengefährdung (S-afety) und bespricht das weitere Vorgehen. Der stechende Knoblauchgeruch, die Bewusstlosigkeit und der massive Speichelfluss lassen eine Alkylphosphatvergiftung mit einem Insektizid wie zum Beispiel E 605 vermuten.

Die Kollegen ziehen über die Einmalhandschuhe noch ein zusätzliches Paar Einmalhandschuhe. Aufgrund der ungeklärten Situation im Schuppen fordern sie neben dem Notarzt auch die Feuerwehr (S-upport) an.

\section{Merke}

Eine Übersicht über rettungsdienstliche Merkhilfen und Akronyme haben wir für Sie auf dem Plakat „Fit für den Einsatz? Das sollten Sie wissen!“ zusammengefasst. Vom SSSS-Schema über die SAMPLER-Anamnese bis zum ABCDE-Schema bietet Ihnen das Plakat eine gute Übersicht zur Vorbereitung auf Ihre Ergänzungsprüfung. Das Plakat können Sie und Ihre Kollegen auf unserer Website bestellen.

\section{ABCDE-Schema}

Penibel vermeidet das Team bei der weiteren Versorgung des Patienten den direkten Hautkontakt zu Speichel und Erbrochenem des Patienten. Schritt für Schritt arbeitet es sich dabei durch das ABCDE-Schema. Wegen der Verdachtsdiagnose erheben die Kollegen den Pupillenstatus. Die Pupillen sind stecknadelkopfgroß - ein weiteres Indiz für die Vergiftung mit einem Alkylphosphat.

A-irway

Die Atemwege drohen aufgrund des massiven Speichelflusses verlegt zu werden. Der Patient wird mithilfe der Absaugpumpe mit einem Absaugkatheter vom Speichel befreit.

\section{B-reathing}

Die Atmung ist jetzt regelmäßig und mit einer Frequenz von 20 Atemzügen pro Minute unauffällig. Der Patient wird in der Seitenlage belassen. Der Brustkorb hebt und senkt sich seitengleich. Beidseits ist ein vesikuläres Atemgeräusch mit leichter Spastik zu hören. Aufgrund des Speichelflusses erhält der Patient den Sauerstoff über eine Nasensonde mit einem moderaten Flow von $5 \mathrm{l} / \mathrm{min}$.

\section{C-Circulation}

Der periphere Puls an der Ateria radialis ist schwach tastbar und mit einer Frequenz von unter 50 Schlägen pro Minute bradykard. Das mittlerweile angelegte EKG zeigt einen Sinusrhythmus und bestätigt die bereits festgestellte Bradykardie. Die Rekapillierungzeit des Nagelbetts liegt unter 2 Sekunden. Der Blutdruck ist mit 90/ $60 \mathrm{~mm} \mathrm{Hg}$ hypoton.

Während ein Teammitglied zwei Ampullen Atropin à $0,5 \mathrm{mg}$ in eine 2-ml-Spritze aufzieht, kümmert sich seine Kollegin um das Legen eines großlumigen venösen Zugangs. Die balancierte Infusionslösung wird zügig infundiert und $1 \mathrm{mg}$ Atropinsulfat appliziert.

Wie zu erwarten, bleibt die Gabe des Antidots ohne nennenswerten Erfolg. Nach wie vor muss das Team in regelmäßigen Abständen die Atemwege von Speichel befreien, die Bradykardie und die Miosis bleiben bestehen. Zur Repetition von Atropinsulfat nutzt das Team eine Ampulle Atropin $0,1 \mathrm{~g}$ in $10 \mathrm{ml}$. Auf eine 10 -ml-Spritze werden $9 \mathrm{ml}$ $\mathrm{NaCl}$ aufgezogen und danach um $1 \mathrm{ml}$ der Atropinsulfatlösung $0,1 \mathrm{~g}$ ergänzt. Der Patient erhält $5 \mathrm{ml}$ der aufgezogenen Lösung.

Der mittlerweile eingetroffene Notarzt verschafft sich einen kurzen Überblick und erhält vom Team eine ausführliche Übergabe. Da sich die Symptome auch nach der erneuten Gabe von 5 mg Atropin nicht gebessert haben, ordnet er eine fraktionierte Gabe in 10-mg-Schritten an. Dazu nutzt das Team die unverdünnte AtropinAmpulle mit $0,1 \mathrm{~g}$ in $10 \mathrm{ml}$.

Nach der Repetition von insgesamt 50 mg Atropinsulfat lässt der Speichelfluss deutlich nach und die Herzfrequenz steigt auf über 60 Schläge pro Minute. Die Pupillen sind nun mittelweit.

\section{Giftnotrufzentrale}

Der Patient ist nun annährend stabil und der Notarzt nutzt die Gelegenheit, um die Giftnotrufzentrale zu kontaktieren. Während das Team den Transport vorbereitet, verschafft sich die Feuerwehr einen Überblick. Feuerwehrleute mit Atemschutz und Chemikalienschutzanzug bringen einen verrosteten Kanister mit der Aufschrift E 605-Staub zum Vorschein. Außerdem haben sie ein Glas mit Resten eines gelbbräunlichen Substanzgemisches gefunden.

\section{D-isability und E-xposure}

Noch vor dem Umlagern des Patienten wird dieser komplett entkleidet. Die Feuerwehr entsorgt die Kleidung. Im Fahrzeug werden die noch fehlenden Untersuchungen ergänzt. Die Ganzkörperinspektion ergibt keinen auffälligen Befund. 


\section{Ehefrau}

Die Fahrerin des Notarzteinsatzfahrzeugs (NEF) lässt sich von der Ehefrau die notwendigen Daten geben. Dabei fällt ihr das bleiche Gesicht der Frau auf. Auf Befragen gibt diese leichten Schwindel, Übelkeit und Kopfschmerzen an. Da die Frau ihren Ehemann aus dem Schuppen gerettet hat, ist eine Intoxikation durch Hautkontakt und Einatmen von giftigen Dämpfen sehr wahrscheinlich. Für den Transport wird ein weiteres Einsatzmittel nachgefordert. Die Symptome bleiben unspezifisch, von einer Antidottherapie wird abgesehen.

\section{Weitere Therapie}

Nach Rücksprache mit der Giftnotrufzentrale verabreicht der Notarzt ergänzend zum Atropin eine Ampulle Toxogonin 250 mg (Obidoxim). Der Patient wird narkotisiert und intubiert und erhält über eine Magensonde $40 \mathrm{~g}$ Aktivkohle zur Giftbindung. In der Klinik wird eine Magenspülung durchgeführt und um eine Hämoperfusion (Dialyse) zur Entfernung der Phosphorsäureester ergänzt.

\section{Der Algorithmus}

Das Insektizid/Akarizid E 605 (Parathion, Thiophos) darf in Europa seit 2002 nicht mehr gehandelt werden. Es wirkt bereits beim Einatmen oder beim Hautkontakt. Unabhängig von diesem Verbot lagern in Bauernhöfen oder bei Kleingärtnern ggf. noch Restbestände, die nicht fachgerecht entsorgt wurden. Wegen der hohen Toxizität erlangte E 605 (E Combi, P-O-X) traurige Berühmtheit bei Selbstmördern, Mördern, Lebensmittel-Erpressern und Menschen, die sich des Nachbarhundes entledigen wollten. Dies ist sicherlich ein Grund dafür, dass für das Pflanzenschutzmittel der Begriff „Schwiegermuttergift“ verwendet wurde.

Artverwandte Stoffe wie Tabun und Sarin wurden jahrzehntelang in der chemischen Kriegsführung als Nervengase eingesetzt. Auch wenn die Stoffe seit 1997 international geächtet sind, tauchen sie immer wieder im Zusammenhang mit Terroranschlägen oder Kriegshandlungen auf.

Alternative Phosphorsäurester mit ähnlicher Wirkungsweise, zum Beispiel Metasystox, Bi 58 oder Perfekthion, sind zwar nicht so toxisch wie E 605, doch können sie heute einfach über das Internet oder in Baumärkten bezogen werden. Ein unsachgemäßer Umgang, insbesondere in suizidaler Absicht, kann auch bei ihnen zu einer lebensbedrohlichen Intoxikation führen. Die Notfallversorgung erfolgt in diesen Fällen genauso wie bei E 605.

Das Team im oben beschriebenen Fall nutzt bei der Versorgung des Patienten den Muster-Algorithmus des
DBRD in der Version 4.0 von 2019 ( $\triangleright$ Abb. 1). Jährlich erfolgt durch ein Expertenteam eine Revision der Algorithmen.

\section{Merke}

Laden Sie sich kostenfrei die Algorithmen des DBRD herunter unter www.dbrd.de. Sollten Sie in Ihrem Rettungsdienstbereich ein abweichendes Konzept nutzen, strukturieren Sie das Fallbeispiel anhand des bei Ihnen eingesetzten Algorithmus und arbeiten Sie die Unterschiede heraus.

\section{Knoblauchgeruch}

Parathion ist eine farblose und fast geruchlose Flüssigkeit. Die in den Handel gebrachten Produkte wurden zur Sicherheit gelbbraun eingefärbt und mit einem stechenden knoblauchartigen Geruch aromatisiert.

\section{Eigenschutz}

Da die Ehefrau den Patienten bereits aus dem Gefahrenbereich gezogen hatte, bleibt dem Team die Entscheidung, ob eine Rettung aus dem Schuppen gefahrlos möglich ist, erspart. Grundsätzlich steht dabei der Eigenschutz des Einsatzpersonals im Vordergrund.

Da das Toxin in erster Linie als Kontaktgift wirkt, vermeidet das Team jeglichen direkten Kontakt zum Patienten und zieht ein zweites Paar Einmalhandschuhe über. Eine Schutzbrille sowie ein Mundschutz sollten den Eigenschutz zwingend ergänzen. Auch sollte die komplette Schutzkleidung getragen werden. Gerade im Sommer beobachtet man häufig, dass Einsatzpersonal in T-Shirts arbeitet.

In unserem Fall besteht besondere Gefahr bei plötzlichem Erbrechen oder bei Hautkontakt mit dem Speichel.

\section{Fünf-Finger-Regel}

Bei der Versorgung einer Intoxikation hilft nach der Beachtung des Eigenschutzes die Fünf-Finger-Regel.

\section{Sicherung der Atmungs- und Kreislauffunktionen}

Lebensrettende Sofortmaßnahmen (z. B. stabile Seitenlage, Freimachen der Atemwege, assistierte oder kontrollierte Beatmung, Herzdruckmassage ...) haben Vorrang.

\section{Wenn möglich, Giftelimination}

Meist ist eine Giftentfernung nicht möglich. Induziertes Erbrechen sollte unterlassen werden und ist, wenn überhaupt, nur beim bewusstseinsklaren Patienten direkt nach der Gifteinnahme sinnvoll (Cave: ätzende oder schaumbildende Substanzen). Klinisch besteht die Möglichkeit einer Magenspülung. 


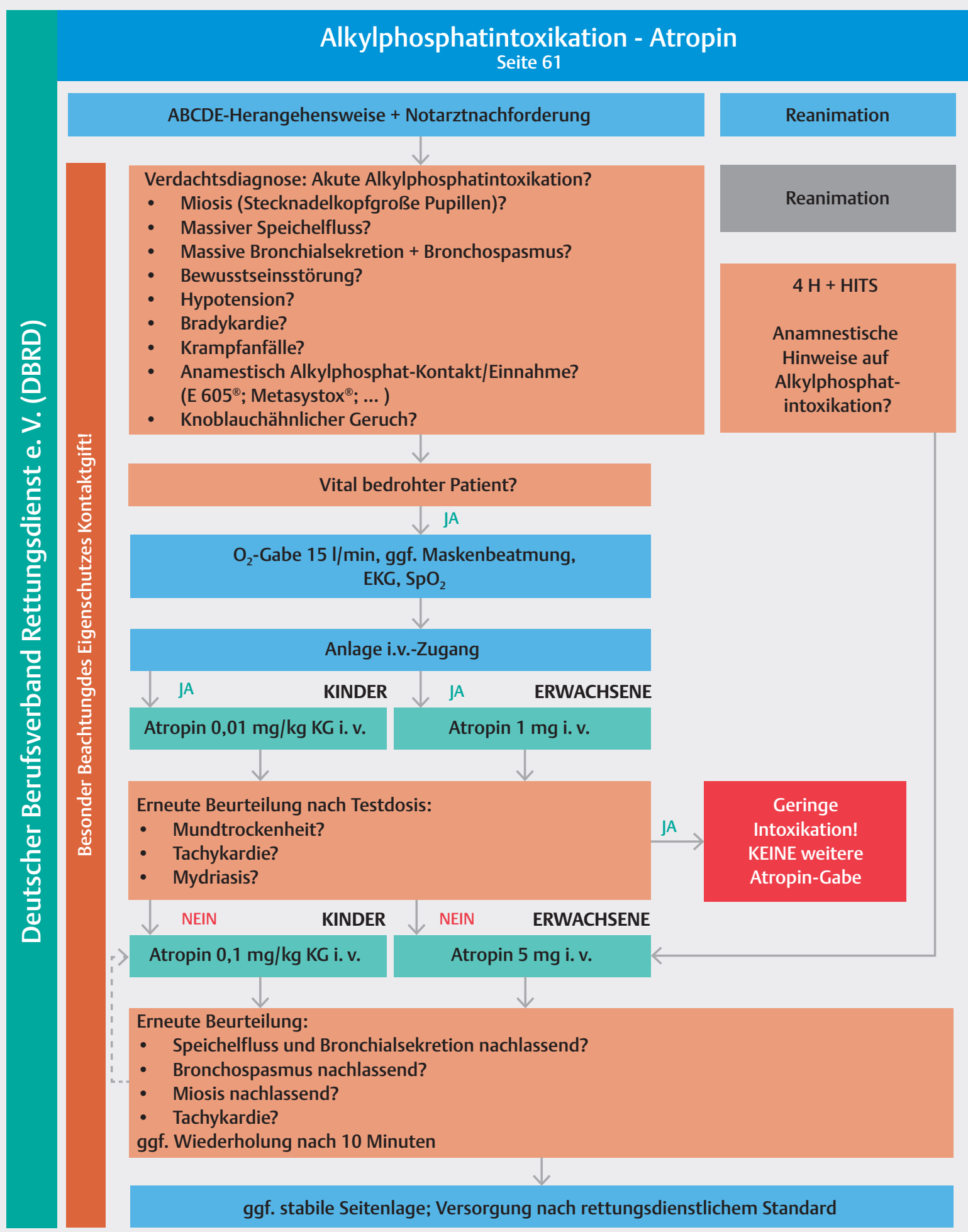

Abb. 1 DBRD-Algorithmus Alkylphosphatintoxikation. Quelle: DBRD Muster-Algorithmen 2019 zur Umsetzung des Pyramidenprozesses im Rahmen des NotSanG - Version 4.0; Deutscher Berufsverband Rettungsdienst e. V. (DBRD)

In jedem Fall sollte eine weitere Aufnahme des Gifts unbedingt vermieden werden (z. B. Entfernen einer Spritze, Retten aus dem Gefahrenbereich, Abwaschen der Haut, Gabe von Medizinaktivkohle ...).

\section{Antidot-Therapie}

Die frühzeitige Behandlung mit einem Antidot kann nur dann erfolgen, wenn Klarheit über das Toxin besteht. 
Eine gute Hilfe bieten dabei die Giftnotrufzentralen/Vergiftungsinformationszentralen. Diese sind 24 Stunden mit speziell ausgebildeten Mitarbeitern besetzt und geben Auskünfte zur Versorgung der Patienten.

\section{Asservierung}

Häufig wird die Asservierung von Substanzen (z. B. leere Medikamentenschachteln, Spritzenbesteck, Substanzreste, Erbrochenes ...) vergessen. Für einen qualitativen und quantitativen Giftnachweis können diese allerdings sehr wichtig sein.

\section{Transport}

Neben einem raschen Transport sollte die Zielklinik möglichst frühzeitig über den Patienten und dessen Verdachtsdiagnose informiert werden. Im besten Fall hat der aufnehmende Arzt/die aufnehmende Ärztin die Zeit bis zum Eintreffen des Rettungswagens genutzt, um sich eingehend über die Gefahren, Risiken und Therapiemöglichkeiten zu informieren.

\section{Sicherung der Atemwege}

Das Team verzichtet auf eine assistierte Beatmung und belässt den Patienten in der stabilen Seitenlage. Dank der seitlichen Lagerung kann der Speichel ungehindert abfließen. Zudem wird der Mundraum des Patienten regelmäßig abgesaugt. Zwar weicht die Oxygenierung mit 5 I Sauersoff über eine Nasensonde vom Algorithmus $\mathrm{ab}$, doch unter den beschriebenen Umständen birgt die Verwendung einer Sauerstoffmaske die Gefahr einer Aspiration.

\section{Atropingabe}

Nach der Anlage eines venösen Zugangs appliziert das Team $1 \mathrm{mg}$ Atropinsulfat. Da eine Wirkung ausbleibt, wiederholt es die Gabe, algorithmenkonform, mit $5 \mathrm{mg}$. Die Gabe von $5 \mathrm{mg}$ Atropinsulfat ist eine kleine Herausforderung. Gerade bei Ungeübten kann es sehr schnell zu Problemen beim richtigen Anmischen der Verdünnung kommen.

\footnotetext{
Merke

Um Fehler zu vermeiden, empfiehlt es sich, die Antidote getrennt im Rettungswagen vorzuhalten. In einer kleinen Tasche können sie idealerweise zusammen mit einer Dosierungsanleitung verwahrt werden.
}

Die richtige Dosierung von Atropinsulfat bei Alkylphosphatvergiftung ist schwierig und orientiert sich in erster Linie an der Pupillenweite. Während es bei versehentlichen Vergiftungen oft schon nach der ersten Gabe von Atropinsulfat zu positiven Effekten kommt, müssen bei massiver Giftexposition (Suizidversuch) mehrere Repetitionen erfolgen.

\section{Transport in die Klinik}

Die weitere Versorgung des Patienten erfolgt gemeinsam mit dem Notarzt unter Beachtung der Informationen der Giftnotrufzentrale.

Besonders hervorzuheben ist die Umsicht der Fahrerin des NEF. Da das Team mit der Versorgung des Patienten beschäftigt ist, kümmert sie sich um das Umfeld und erkennt die Transportindikation für die Ehefrau des Patienten.

\section{Prüfungsfragen}

\section{NOTFALLMEDIZIN}

Erläutern Sie kurz die Wirkungsweise von Alkylphosphaten im synaptischen Spalt und beschreiben Sie die Wirkung von Atropinsulfat und Obidoxin.

Wirkung von Acetylcholin (ACh):

ACh ist ein Neurotransmitter ( $\triangleright$ Abb. 2) an der motorischen Endplatte und vermittelt die Kontraktion der Skelettmuskulatur. Daneben wirkt es als Signalstoff im sympathischen und parasympathischen Nervensystem. Kommt es zu einer Ausschüttung von ACh aus einem Vesikel in den synaptischen Spalt (a.), dockt ACh als Agonist an einen entsprechenden Rezeptor an (b.). Am Rezeptor führt ACh zu einer Öffnung der Natriumkanäle (c.) mit der Folge des vermehrten Natriumeinstroms in die Zelle; es kommt zur Depolarisation. Das ACh wird durch die Acetylcholinesterase im synaptischen Spalt in Cholin und Essigsäure gespalten, im Endköpfchen wieder zu ACh synthetisiert und im Zytoplasma in Vesikeln gespeichert.

Hemmung der Acetycholinesterase durch E 605:

Das Alkylphosphat E 605 bindet die Acetylcholinesterase ( $\triangleright$ Abb. 3) und verhindert dadurch deren Abbau am Rezeptor (a.). Es kommt zu einer andauernden Depolarisation (b.).

Wirkung von Atropinsulfat:

Atropinsulfat konkurriert mit ACh um die Bindung am Rezeptor ( $\triangleright$ Abb.4). Als kompetitiver Antagonist bleibt bei einer Bindung die biochemische Wirkung jedoch aus (a.). Da das Alkylphosphat E 605 im Gegensatz zu Atropinsulfat nur unzureichend abgebaut wird, hält die Antidotwirkung von Atropinsulfat nur vorrübergehend an und macht eine Repetition erforderlich.

Wirkung von Obidoxim:

Obidoxim ( $\vee$ Abb. 5 ) reaktiviert (a.) die blockierte Acetylcholinesterase, indem sie sich an die Phosphatgruppe des Toxins bindet (b.). Die Verbindung wird über die Nieren ausgeschieden. 


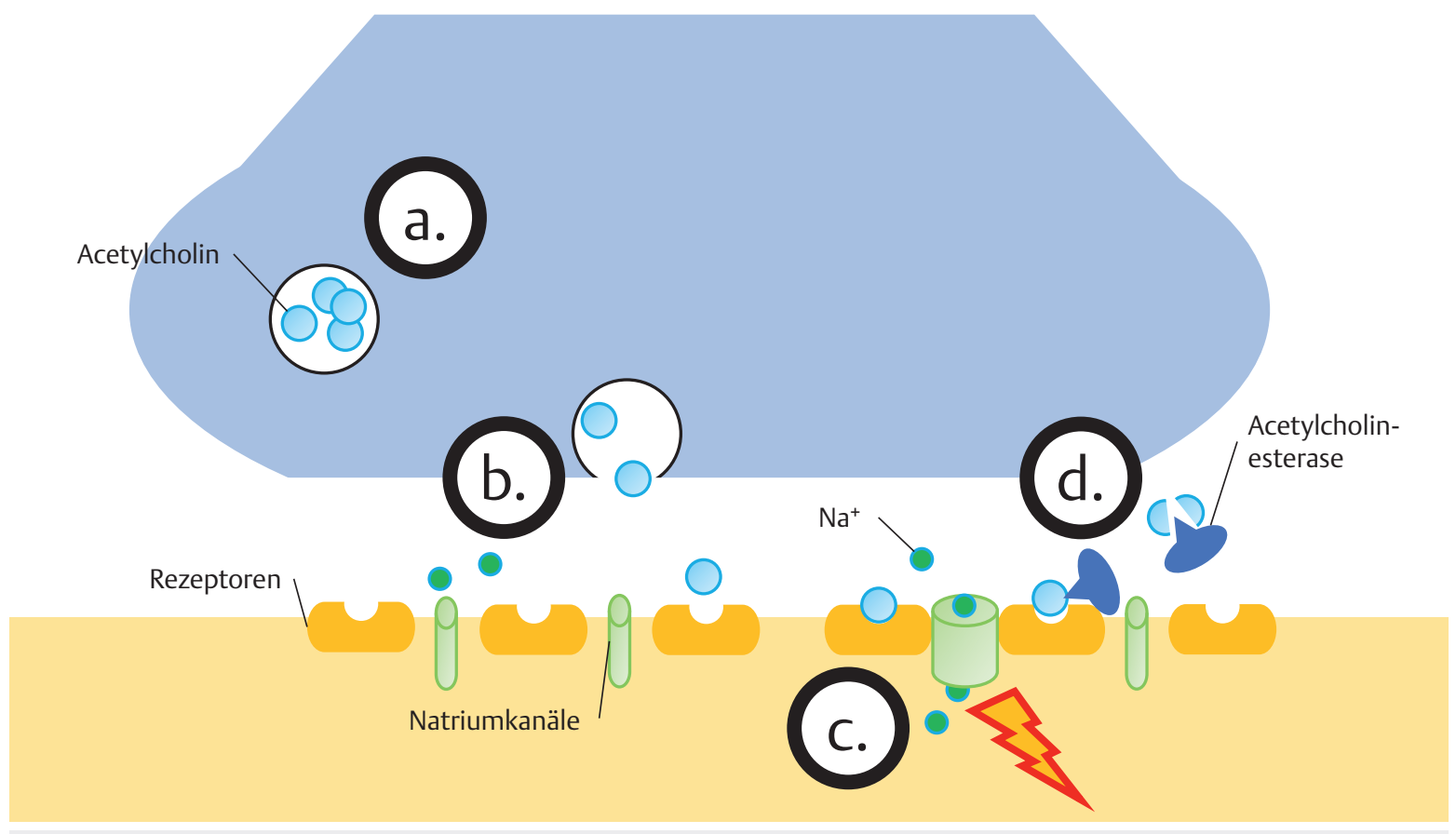

- Abb.2 Physiologische Reaktion von Acetylcholin an der motorischen Endplatte.

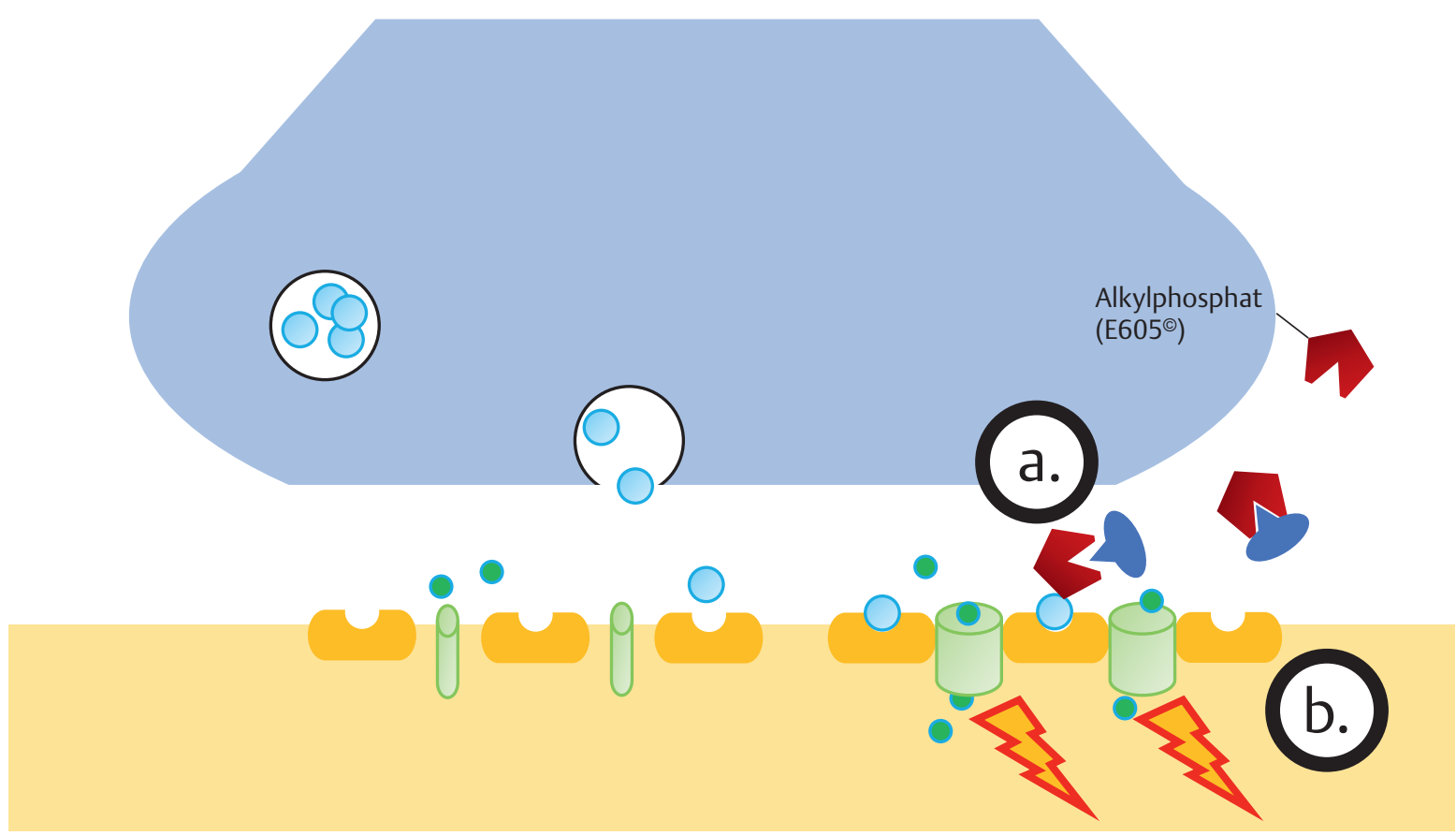

- Abb. 3 Hemmung der Acetycholinesterase durch E 605.

\section{Mögliche weitere Fragen:}

- Beschreiben Sie typische Symptome bei einer Vergiftung mit Alkylphosphat.

- Welche Maßnahmen stehen Ihnen zur Atemwegssicherung zur Verfügung?

- Beschreiben Sie die Therapie bei einem fünfjährigen Kind mit einem Körpergewicht von $19 \mathrm{~kg}$.

\section{KOMMUNIKATION}

? Was versteht man unter dem Eisbergmodell nach Paul Watzlawick?

Paul Watzlawick nutzt die Metapher des ursprünglichen Eisbergmodels von Sigmund Freud im Rahmen der Kommunikation. Er behauptet, dass im zwischenmensch- 


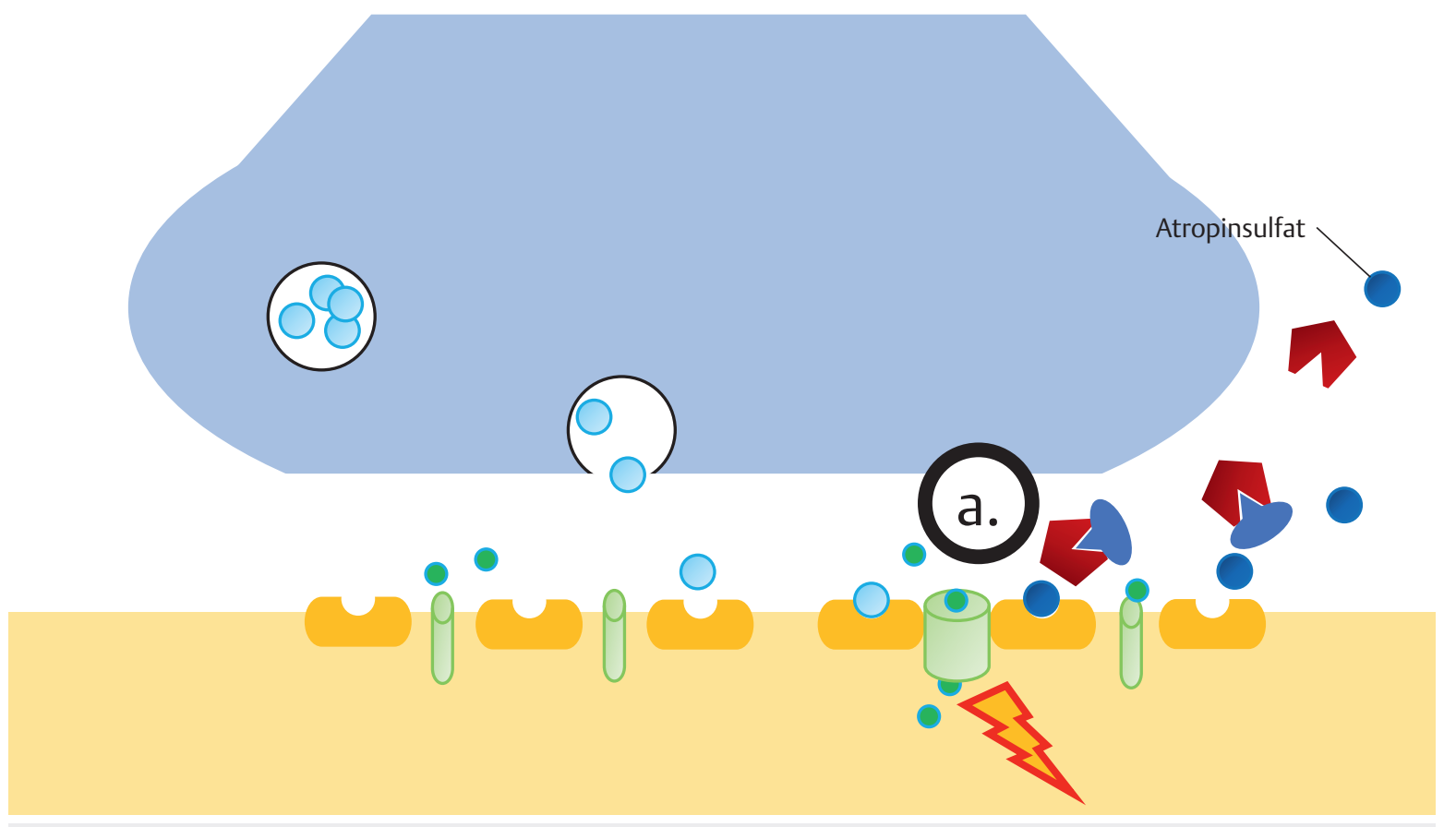

Abb. 4 Wirkung von Atropinsulfat.

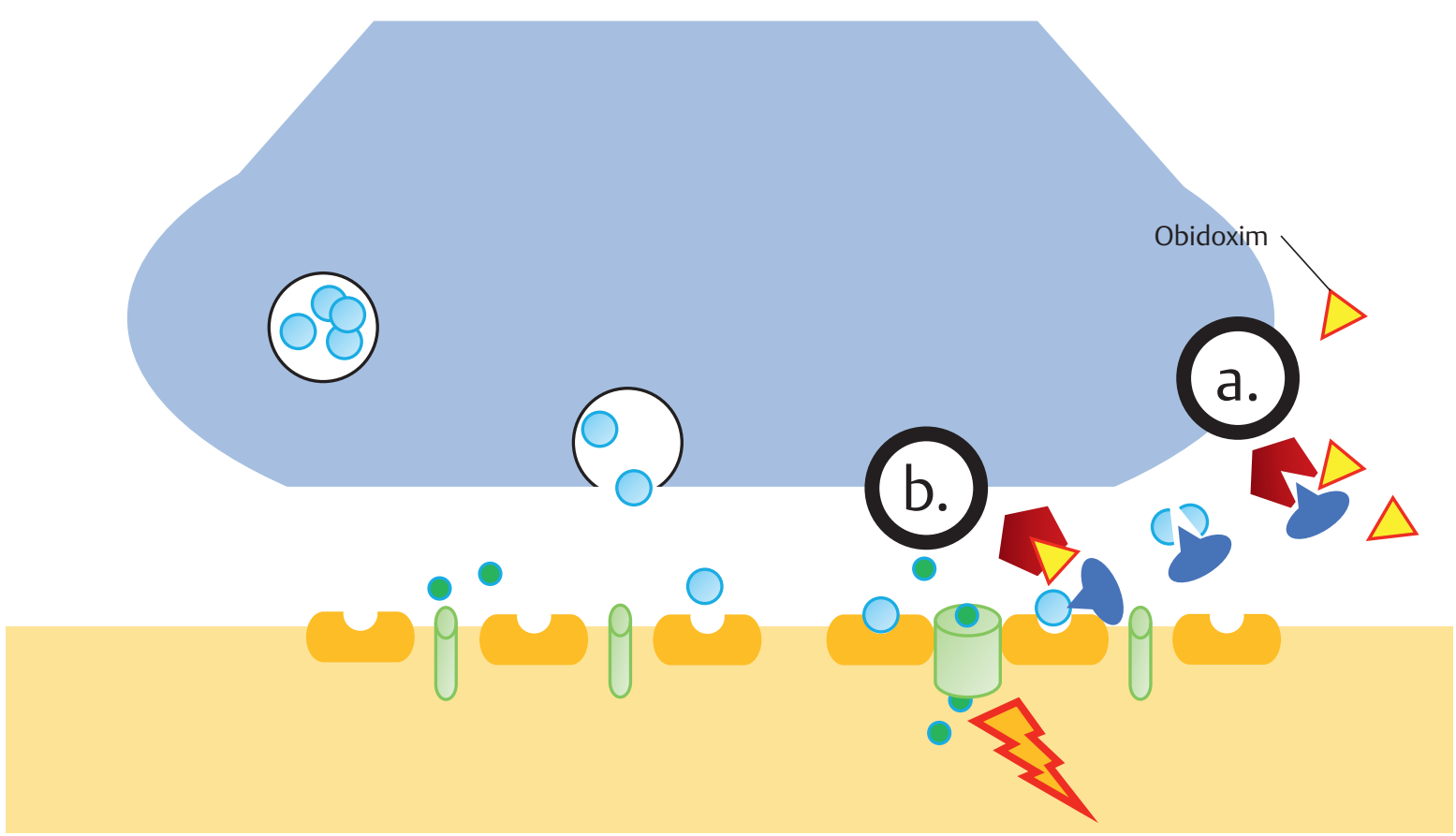

- Abb. 5 Wirkung von Obidoxim.

lichen Kommunikationsverhalten sich lediglich $20 \%$ auf der Sachebene (Zahlen, Daten und Fakten) abspielen, während der weitaus größte Teil (80\%) auf der Beziehungsebene (Instinkt, Gefühle, Ängste, Triebe, Erfahrungen, Traumata) geschieht.

\section{Mögliche weitere Fragen:}

- Beschreiben Sie mögliche Ängste der Frau des Patienten nach dessen Suizidversuch und reflektieren Sie ihre Bedürfnisse.

- Welche Kommunikationsarten lassen sich unterscheiden? 


\section{RAHMENBEDINGUNGEN}

Sie sind mit ihrem Rettungswagen zum Transport der Ehefrau angefordert worden. Gleich zu Beginn schildert Ihnen Ihre Patientin, dass sie in keinem Fall mitkommen könne. Im Haus schliefen noch ihre zwei Kinder (6 Monate und 5 Jahre) in ihren Bettchen. Beschreiben Sie mögliche Lösungswege.

Ein relativ einfacher Weg wäre die Kinderbetreuung durch Verwandte, Freunde oder gute Bekannte. Bis zu deren Eintreffen könnte die Zeit mit vertrauensvollen Nachbarn überbrückt werden. Ist dies nicht möglich, kann eine Betreuung auch über das zuständige Jugendamt organisiert werden. Steht keine der genannten Möglichkeiten zeitnah zur Verfügung, ist mit der Klinik zu klären, ob diese, zumindest für den Übergang, die Kinder mit der Frau stationär aufnehmen könnte.

\section{Mögliche weitere Fragen:}

- Bewerten Sie für das oben beschriebene Fallbeispiel das einsatztaktische Vorgehen der Feuerwehr.

- Hätten Sie sich für eine schnelle Rettung des Patienten aus dem Schuppen entschieden, wenn dieser nicht bereits durch seine Ehefrau gerettet worden wäre? Begründen Sie Ihre Antwort.

\section{KOMMENTAR}

von Thomas Semmel, Notfallsanitäter, Vorstandsmitglied des DBRD

„Alle Dinge sind Gift und nichts ist ohne Gift. Allein die Dosis macht, dass ein Ding kein Gift ist." So oder so ähnlich hat Paracelsus bereits im 15. Jahrhundert die Möglichkeit einer Vergiftung beschrieben. Schaut man sich die hochinteressanten Internetseiten der deutschen Giftnotrufzentralen an, so stellt man fest, dass Vergiftungen gar nicht so selten sind.

Max von Clarmann, ein Toxikologe aus München, hat vor über 20 Jahren zum Thema Vergiftungen 10 Gebote aufgestellt. Eines dieser Gebote hat auch heute noch sehr große Bedeutung, nämlich: im Einsatz auch immer an die Möglichkeit einer Vergiftung zu denken. Wie wichtig das ist, zeigt auch der oben beschriebene Fall.

Auch wenn Intoxikationen mit Alkylphosphaten zu den eher seltenen Einsatzsituationen zählen, ist es wichtig zu wissen, wie die betroffenen Patienten fachgerecht versorgt werden müssen. Und es geht dabei leider nicht nur um den vergessenen Rest eines Pflanzenschutzmittels im Schuppen: Leider gibt es Alkylphosphate, die, wie von den Autoren beschrieben, als chemische Kampfstoffe eingesetzt wurden und möglicherweise noch werden. Im Jahr 1995 haben Mitglieder einer Sekte einen Anschlag auf die U-Bahn in Tokio verübt. Es kam zu mehreren Todesfällen und zu tau- senden Verletzten - man mag sich ein solches Szenario überhaupt nicht vorstellen.

Der DBRD hat auf der Grundlage der vorhandenen Literatur einen Algorithmus zur Behandlung von Patienten mit einer Alkylphosphatintoxikation erstellt. Neben den Muster-Algorithmen bietet der DBRD auch den international anerkannten und zertifizierten Advanced-Medical-Life-Support-Kurs (AMLS) an. Inhalte des 2-tägigen Kurses sind die Beurteilung und Behandlung von Patienten mit internistisch-neurologischen Akuterkrankungen; dazu gehört auch die Behandlung von Vergiftungen. Weitere Informationen dazu finden Sie unter www.amls.de.

\section{Interessenkonflikt}

Die Autoren geben an, dass kein Interessenkonflikt besteht.

\section{Autorinnen/Autoren}

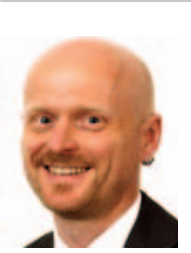

\section{Rico Kuhnke}

MA. Gesamtschulleiter der DRK-Landesschule Baden-Württemberg. Er war viele Jahre als Lehrrettungsassistent tätig und hat sein pädagogisches Masterstudium berufsbegleitend abgeschlossen. Er ist Notfallsanitäter und Mitherausgeber von retten!

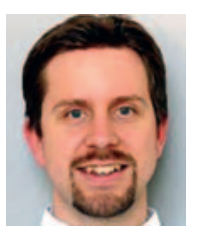

\section{Thomas Ahne}

Dr. med. Facharzt für Anästhesiologie mit Zusatzbezeichnung Notfallmedizin. Der Mitherausgeber von retten! ist klinisch in der internistischen Intensivmedizin des Universitätsklinikums Freiburg tätig sowie präklinisch, boden- wie luftgebunden, in Deutschland, Österreich und der Schweiz.

\section{Korrespondenzadresse}

\author{
Rico Kuhnke \\ Schulleiter \\ Deutsches Rotes Kreuz \\ Landesschule Baden-Württemberg \\ Karl-Berner-Str. 6 \\ 72295 Pfalzgrafenweiler \\ r.kuhnke@drk-Is.de
}

Bibliografie

DOI https://doi.org/10.1055/a-0978-9323

retten 2020; 9: 16-23

(c) Georg Thieme Verlag KG, Stuttgart · New York

ISSN 2193-2387 


\section{ERRATUM}

Alkylphosphatintoxikation - Das sollten Sie wissen für die Ergänzungsprüfung

Kuhnke R, Ahne T. retten! 2020; 09: 16-23. DOI: 10.1055/a-0978-9323

Der Artikel wurde gemäß Erratum vom 13.05.2021 (Hochladedatum) geändert. In Abb. 1 waren die Dosisangaben für Atropin bei Kindern vertauscht. Korrekt werden zuerst 0,01 mg/kg KG i.v. verabreicht, im zweiten Schritt 0,1 mg/kg KG. 